Journal of Social Sciences 1 (4): 246-247, 2005

ISSN 1549-3652

(c) 2005 Science Publications

\title{
Survival in the Current Healthcare Environment How Close Are We to Socialized Medicine?
}

\author{
Eldo E. Frezza \\ Division of General Surgery, Department of Surgery, Texas Tech University Health Sciences Center
}

\begin{abstract}
In the 1980's, some hospitals established internal agencies to regulate cash flow and capital expense. State agencies promoted the evolution of healthcare facilities into more business-like organizations. Attention to the following matters will reduce hospital costs: 1) prevent or reduce nosocomial infections; 2) treat diseases at a stage amenable to outpatient therapy; 3) obtain complete information about the policies of each HMO, adhering to them as closely as possible; and 4) keep comprehensive medical records, both to justify every admission and to penalize physicians who do not fully justify admission. Co-payments have increased from hundreds to thousands of dollars: the first $\$ 1,000$ to $\$ 5,000$ often comes from the patient's pocket. New technology and drugs must be paid for by premium increases. Hospital costs have increased beyond premium increases. The situation will probably explode unless something happens. Socialized medicine will solve the problems of nonpayment by patients, but it will affect the middle class who are struggling with economics and will put them in worsening financial status, while the people with more income will end up paying out of pocket to get the care they need.
\end{abstract}

Key words: Healthcare, social medicine, capitation system, prospective payment, DRG, cost reimbursement

\section{INTRODUCTION}

The healthcare economy has fluctuated greatly in the United States. State agencies promoted the evolution of healthcare facilities into more business-like organizations. Prior to the introduction of fee for service at this time, cost accounting was almost nonexistent in the healthcare industry.

Justin Ford Kimbalo, a member of Baylor University's board of trustees, began the first insurance company in the United States. His founding of Blue Cross in the 1930's arose out of concern about the effect of large numbers of patients who were not paying their hospital bills. At the time, most hospitals were non-profit corporations run by charitable organizations that Kimbalo thought should be protected from financial risks; providers were paid by a system called cost reimbursement ${ }^{[1]}$.

Eric Kaiser, who had a contract to build ships for the war effort, formed the first health maintenance organization (HMO) in the 1940's. In order to recruit employees without violating wage controls, he began offering them health benefits. Healthcare providers were paid prospectively via a system called capitation.

The introduction of the prospective payment system in the 1980's regulated healthcare providers and, in some instances, encouraged waste of resources.
Prospective payment as a replacement for cost reimbursement: Under a prospective payment system, the insurance company negotiates a fixed price for a set of medical goods and services prior to the onset of the patient's illness. Prospective payment better controls cost than does cost reimbursement, but it increases financial risks to the hospital and physician. Under cost reimbursement, payment is made according to the length of stay. Therefore, longer hospital stays increase hospital reimbursement. Hospitals were not concerned with how long the patient stayed in the hospital.

Most health insurances assumed the role of intermediary. Insurance companies simply passed the additional cost to the employer, and the employer in return increased the premiums. Employers were essentially unaffected by the decision to leave an employee in the hospital for unneeded days, as they would transfer the cost back to the employee through higher premiums. Therefore, cost reimbursement provided few incentives for cost control ${ }^{[2]}$.

Capitation system: Under capitation, providers receive a fixed amount per patient per month to provide specific services, whether or not patients actually become sick. The system would supposedly provide an incentive to keep the patient well and encourage the use of the most cost-effective resources to get them better.

Corresponding Author: Eldo Ermenegildo Frezza, MD, MBA, Department of Surgery, Division of General Surgery, Texas Tech University Health Sciences Center, MOP Building, Suite 380, $35029^{\text {th }}$ Street, Lubbock, Texas 79415, Tel: 806-743-2460 X263, Fax: 806-743-2113 
Treatments given the patient were emphasized: providers lost money by keeping patients in the hospital longer than needed or by underutilizing cheaper outpatient services. Bonuses for the especially efficient utilization of resources furthered this trend; the downside was fraud, poor quality of care, and underassessment of disease processes ${ }^{[2]}$.

DRG: Diagnosis Related Groups (DRG's), proved the most effective means of controlling costs for hospitals. Reimbursement for admission is fixed, the financial incentive for hospitals is to provide only those services needed to cure the patients as rapidly as possible.

\section{Recommendations for economic survival in the} current healthcare environment: Generalized processes for reducing hospital and HMO costs under capitation include:

1. Avoiding unnecessary hospital admissions

2. Decreasing the length of stay in the hospital

3. Treating patients on an outpatient basis whenever feasible

4. Encouraging preventative care as much as possible.

Attention to the following matters will further reduce hospital costs:

1. Prevent or reduce nosocomial infections

2. Detect diseases to treat as outpatient

3. Obtain complete information about the policies of each HMO, adhering to them as closely as possible

4. Keep comprehensive medical records, both to justify every admission and to penalize physicians who do not fully justify admission.

For the physician, recommendations are similar, HMO's may further control costs incurred by physicians by restricting the number of cancer cases treated on an inpatient and outpatient basis or reducing the average amount of time spent with patients and pushing physicians to see more patients per hour. Salaries and bonuses are often based on efficiency with resources.

For employers, a number of steps can be taken to reduce costs. By aggressively negotiating prices with HMO's, instituting wellness programs at work, giving bonus incentives to decrease work-related injuries, and giving bonuses for increasing healthy lifestyles, employers will better profit under the current system $^{[2,3]}$.
Healthcare inflation under capitation: The cost of healthcare is rising. Premiums for every family have increased in the last 20 years. Most insurance companies are now turning into "80/20" or "70/30" plans, where patients pay $20 \%$ or $30 \%$ of the bill; some insurance companies are even moving to a "50/50" plan.

Co-payments have increased from hundreds to thousands of dollars: the first $\$ 1,000$ to $\$ 5,000$ often emerges from the patient's pocket. New technology and drugs, beneficial to life and health, are paid for by premium increases. Hospitals bear a particular burden from the nursing shortage-wages of nurses have increased beyond premium increases.

A future of socialized medicine: Many people believe the escalating costs of healthcare will yield socialized medicine. As more persons become uninsured, due to the high cost of insurance, or uninsurable, due to preexistent health conditions, as society ages, and as technology advances, private health insurance will become less prevalent ${ }^{[2]}$. As government funding becomes more prevalent, socialized medicine, however demonized it may be, becomes more of a reality.

Unfortunately, outside of the United States in statebased healthcare, patients often have to wait nine to 10 months for a mammogram. Those wanting faster care must go to private clinics, which charge far more to the patient than is the case with private insurance. Socialized medicine will solve the problems of nonpayment by patients, but it will affect the middle class who are struggling with economics and will put them in worsening financial status, while the people with more income will end up paying out of pocket to get the care they feel they deserve.

\section{REFERENCES}

1. Code Blue, 2002. Third Edition. McDermott RE, Stocks KD. Traemus Books.

2. Frezza, E.E., 2006. The six stages of the healthcare economy-is socialized medicine at the door? A historical review. J. Social Sci., 2006;2:---.

3. Frezza, E.E. and M. Chiriva-Internati, 2005. The evolution of today's healthcare economy. J. Social Sci.. 1: 39-40. 